PROCEEDINGS OF THE

AMERICAN MATHEMATICAL SOCIETY

Volume 135, Number 10, October 2007, Pages 3249-3254

S 0002-9939(07)08836-3

Article electronically published on June 20, 2007

\title{
ON THE GROWTH OF THE NUMBER OF PERIODIC POINTS FOR SMOOTH SELF-MAPS OF A COMPACT MANIFOLD
}

\author{
GRZEGORZ GRAFF AND JERZY JEZIERSKI
}

(Communicated by Jane M. Hawkins)

\begin{abstract}
Let $f$ be a continuous self-map of a smooth compact connected and simply-connected manifold of dimension $m \geq 3$. We show that in the homotopy class of $f$ there is a $C^{1}$ map with less then $r$ periodic points, up to any given fixed period $r$.
\end{abstract}

\section{INTRODUCTION}

Shub and Sullivan (cf. [SS] ) considered a smooth self-map $f$ of a compact manifold $M$ such that fixed points of $f^{n}$ are isolated. Under the condition that the sequence of Lefschetz numbers $\left\{L\left(f^{n}\right)\right\}_{n=1}^{\infty}$ is unbounded, they proved that $f$ has an infinite number of periodic points with distinct minimal periods. In particular all smooth self-maps of $S^{m}$ of degree $d$, with $|d|>1$, have this property. The natural question arises: what is the growth rate of the number of periodic points (with period not greater than a prescribed $r$ )? There are open sets of $C^{k}$ diffeomorphisms where generic (residual) maps have arbitrary fast growth of the number of periodic points (cf. $[\mathrm{K}]$ ). As the growth of unbounded Lefschetz numbers is exponential, Shub and Sullivan conjectured that for any given smooth map $f$ the growth of the number of periodic points is also at least (asymptotically) exponential, namely,

$$
\limsup _{r \rightarrow \infty} \frac{\log \# \operatorname{Fix}\left(f^{r}\right)}{r} \geq \limsup _{r \rightarrow \infty} \frac{\log \left|L\left(f^{r}\right)\right|}{r} .
$$

The main result of this paper is related to the Shub-Sullivan Conjecture and is the following.

Theorem 1.1. Let $f: M \rightarrow M$ be a continuous self-map of a smooth compact connected and simply-connected manifold $M$, $\operatorname{dim} M \geq 3$. Let $r \in \mathbb{N}, r \geq 2$. For $M$ with nonempty boundary we assume additionally that $f$ has no periodic points on the boundary.

Then $f$ is homotopic to a smooth map g satisfying

$$
\#\left(\bigcup_{i \leq r} \operatorname{Fix}\left(g^{i}\right)\right) \leq r-1 .
$$

Received by the editors March 30, 2006 and, in revised form, June 30, 2006.

2000 Mathematics Subject Classification. Primary 37C25, 55M20; Secondary 37C05.

Key words and phrases. Periodic points, $C^{1}$ maps, indices of iterations, Nielsen number.

This research was supported by KBN grant No 1 P03A 03929.

(C)2007 American Mathematical Society 
Of course the above theorem does not contradict the Shub-Sullivan Conjecture, since our methods do not control what happens in the periods higher than a prescribed $r$. It only states that for each map $f$ the growth of the number

$$
h_{r}[f]=\min _{g} \#\left(\bigcup_{i=1}^{r} \operatorname{Fix}\left(g^{i}\right)\right),
$$

where $g$ runs over the set of smooth maps homotopic to $f$, is not faster than linear. By Theorem 1.1 we may generate examples of smooth maps with an unbounded sequence of Lefschetz numbers with a relatively small set of periodic points, up to any given fixed period $r$. The growth of

$$
c_{r}(f)=\#\left(\bigcup_{i=1}^{r} \operatorname{Fix}\left(f^{i}\right)\right),
$$

as well as \# Fix $\left(f^{r}\right)$, still remains unknown. On the other hand the growth of $c_{r}(f)$ must be at least linear (with the coefficient depending on $f$ and a manifold), which follows from $[\mathrm{BaBo}$ :

Remark 1.2. Babenko and Bogatyi proved that for $f$, a self-map of an $m$-dimensional smooth compact manifold $M$, such that $\left\{L\left(f^{n}\right)\right\}_{n=1}^{\infty}$ is unbounded, the following inequality holds:

$$
O(f, r) \geq \frac{r-N_{0}}{D \cdot 2^{[(m+1) / 2]}},
$$

where $O(f, r)$ is the number of periodic orbits of period no greater than $r, D=$ $\operatorname{dim} H_{*}(M, \mathbb{Q})$ and $N_{0}$ is a natural number.

As a consequence, the inequality $c_{r}(f)>O(f, r)$ gives the linear estimate from below for $c_{r}(f)$.

\section{Preliminary Results}

2.1. Fixed point indices of iterations. Let $f$ be a continuous self-map of a compact smooth manifold. We deal with the problem what is the least number of periodic points of a smooth map homotopic to $f$. It appears that the forms of local fixed point indices of iterations of $C^{1}$ maps are crucial for studying the possibility of cancelling the periodic points in the homotopy class.

In 1983 A. Dold (cf. [D1]) gave some necessary conditions (called Dold relations) which must be satisfied by any sequence of indices of iterations. Namely, let $f$ : $U \rightarrow X$, where $U$ is an open subset of a finite polyhedron $X$ (or more generally, of a Euclidean Neighborhood Retract).

We denote inductively $U_{0}=U, U_{n+1}=f^{-1}\left(U_{n}\right)$. We assume that the fixed point set $\operatorname{Fix}\left(f^{n}\right)=\left\{x \in U_{n}: f^{n}(x)=x\right\}$ is compact for each $n \in \mathbb{N}$. In such a situation the fixed point index $\operatorname{ind}\left(f^{n}\right)=\operatorname{ind}\left(f^{n}, U_{n}\right)$ is well-defined (cf. D2]). Dold proved that the sequence of fixed point indices $\left\{\operatorname{ind}\left(f^{n}\right)\right\}_{n=1}^{\infty}$ must satisfy the following congruences: for each $n \in \mathbb{N}$,

$$
\sum_{k \mid n} \mu(n / k) \operatorname{ind}\left(f^{k}\right) \equiv 0 \quad(\bmod n),
$$

where $\mu$ is the classical Möbius function, i.e., $\mu: \mathbb{N} \rightarrow \mathbb{Z}$ is defined by three properties: $\mu(1)=1, \mu(k)=(-1)^{r}$ if $k$ is a product of $r$ different primes, $\mu(k)=0$ otherwise. 
Let $f$ be a $C^{1}$ map with an isolated fixed point $x_{0}$. Then there are further strong restrictions, except for the Dold relations, on the form of the local indices $\left\{\operatorname{ind}\left(f^{n}, x_{0}\right)\right\}_{n=1}^{\infty}$ (cf. [SS], [CMPY]).

A complete description of sequences which can be realized as indices at an isolated fixed point in $\mathbb{R}^{3}$ is given in GNP. For the sake of this paper we need the existence of smooth maps which have sequences of local indices of iterations in $\mathbb{R}^{m}$, $m \geq 3$, following two special patterns. The constructions of such maps may be found by the reader in GNP (Theorem 3 cases (A) and (D)).

Lemma 2.1. Let $a_{1}, a_{2}$ and $a_{d}, d>1$ be arbitrarily given integers and let $x_{0} \in \mathbb{R}^{m}$, $m \geq 3$. Then there exist: an open neighborhood $U$ of $x_{0}$ and $C^{1}$ maps $f_{a_{1}, a_{2}} ; f_{a_{d}}$ : $U \rightarrow \mathbb{R}^{m}$, such that $x_{0}$ is an isolated fixed point for each iteration and

$$
\begin{gathered}
\operatorname{ind}\left(f_{a_{1}, a_{2}}^{n}, x_{0}\right)=\left\{\begin{array}{cc}
a_{1} & \text { if } 2 \not x n, \\
a_{1}+2 a_{2} & \text { if } 2 \mid n,
\end{array}\right. \\
\operatorname{ind}\left(f_{a_{d}}^{n}, x_{0}\right)=\left\{\begin{array}{cc}
0 & \text { if } d \not 1 n, \\
a_{d} d & \text { if } d \mid n .
\end{array}\right.
\end{gathered}
$$

Remark 2.2. Let us emphasize that maps with the indices of iterations of the form $(*)$ and $(* *)$ do not exist in $\mathbb{R}^{m}$ for $m<3$ (cf. $\mathrm{BaBo}$ ); i.e., 3 is the lowest dimension in which such types of sequences may be realized as local indices of iterations of a $C^{1}$ map.

2.2. Adding and cancelling periodic points in a homotopy class. In the proof of Theorem 1.1 we will also need two statements, proved by the methods of Nielsen Periodic Point Theory in JJe (cf. also [JM]), which allow one to add or remove some periodic points in a homotopy class. We assume in this section that $M$ is a compact connected and simply-connected manifold of dimension at least 3 .

The following theorem (Creating Procedure) enables one to create an additional orbit in the homotopy class of $f$, by a homotopy $f_{t}$ which is constant near periodic points of $f$ (up to the given period $r$ ) and such that $f_{1}^{n}$ near the created $n$-orbit may be given by an arbitrarily prescribed formula. More precisely:

Theorem 2.3 ([J], Theorem 3.3). Given numbers $n, r \in \mathbb{N}, n \mid r$ and a continuous map $f: M \rightarrow M$, where $\operatorname{dim} M \geq 3$, such that $\operatorname{Fix}\left(f^{r}\right)$ is finite and a point $x_{0} \notin \operatorname{Fix}\left(f^{r}\right)$, then there is a homotopy $\left\{f_{t}\right\}_{0 \leq t \leq 1}$ satisfying:

(1) $f_{0}=f$.

(2) $\left\{f_{t}\right\}$ is constant in a neighborhood of $\operatorname{Fix}\left(f^{r}\right)$.

(3) $f_{1}^{n}\left(x_{0}\right)=x_{0}$ and $f_{1}^{i}\left(x_{0}\right) \neq x_{0}$ for $i=1, \ldots, n-1$.

(4) The map $f_{1}^{n}$ is given near $x_{0}$ by an arbitrarily prescribed formula $\phi$ with the property $\phi^{\frac{r}{n}}(x)=x \Longleftrightarrow x=x_{0}$.

(5) The orbit $x_{0}, f_{1}\left(x_{0}\right), \ldots, f_{1}^{n-1}\left(x_{0}\right)$ is isolated in $\operatorname{Fix}\left(f_{1}^{r}\right)$.

The next lemma enables one to cancel some subsets of periodic points with indices of iterations equal to zero.

Lemma 2.4 ([Je], Lemma 5.2). Let $f$ be a continuous self-map of $M$. Suppose that $S \subset \operatorname{Fix}\left(f^{r}\right)$ satisfies:

(1) $S$ is finite and $f$-invariant, i.e., $f(S)=S$.

(2) $\operatorname{Fix}\left(f^{r}\right) \backslash S$ is compact.

(3) $\operatorname{ind}\left(f^{n}, \operatorname{Fix}\left(f^{n}\right) \backslash S\right)=0$ for all $n \mid r$. 
Then there is a homotopy $f_{t}$, starting from $f_{0}=f$, constant near $S$ and such that $\operatorname{Fix}\left(f_{1}^{r}\right)=S$.

We are ready to prove our main theorem. The brief scheme of the proof is the following. We will use an inductive procedure: at the inductive step we add a single fixed point by the Creating Procedure (Theorem 2.3), taking as $\phi$ an appropriate realization from Lemma 2.1 i.e., inserting locally a $C^{1}$ map with a fixed point having indices following the given pattern. This is done in such a way that at the end of the homotopy the other periodic points have indices equal to zero, so may be removed by Lemma 2.4.

\section{Proof of Theorem 1.1}

We use induction for $r \geq 2$. Let $r=2$. We assume that $\operatorname{Fix}\left(f^{2}\right)$ is finite, since otherwise we may approximate $f$ by a smooth map which has a finite number of fixed and 2-periodic points by the Kupka-Smale theorem.

Next we create an additional fixed point $z_{2} \notin \operatorname{Fix}\left(f^{2}\right)$ by the Creating Procedure $(n=1)$, deforming $f$ to $\bar{f}_{2}$ in such a way that $\bar{f}_{2}=f_{a_{1}, a_{2}}$ near $z_{2}$ with $a_{1}=\operatorname{ind}(f)$, $a_{2}=\frac{1}{2}\left(\operatorname{ind}\left(f^{2}\right)-\operatorname{ind}(f)\right)$ (cf. item 4 of Theorem 2.3 and $(*)$ of Lemma 2.1). Notice that by the Dold relations (cf. Formula 2.1) $a_{2}$ is an integer. As a result, by Theorem 2.3, $\bar{f}_{2}$ is homotopic to $f$ and

$$
\begin{gathered}
\operatorname{ind}\left(\bar{f}_{2}, z_{2}\right)=a_{1}=\operatorname{ind}(f), \\
\operatorname{ind}\left(\bar{f}_{2}^{2}, z_{2}\right)=a_{1}+2 a_{2}=\operatorname{ind}\left(f^{2}\right) .
\end{gathered}
$$

Now $\bar{f}_{2}, S=\left\{z_{2}\right\}$ and $r=2$ satisfy the assumptions (1)-(3) of Lemma 2.4 hence $\bar{f}_{2}$ is homotopic, rel. a neighborhood of $z_{2}$, to a map $f_{2}$ with $\operatorname{Fix}\left(f_{2}^{2}\right)=\left\{z_{2}\right\}$. The map $f_{2}$ is smooth in some neighborhood $U_{2}$ of $z_{2}$ and $f_{2}^{2}$ has no fixed points outside $U_{2}$. Thus, if $f_{2}$ were not smooth as the global self-map of $M$, we could approximate it by a smooth map, and then use a homotopy constant on $U_{2}$, without adding new $r$-fixed points $(r=1,2)$ in the compact set $M \backslash U_{2}$.

Now we assume that $\bigcup_{i<r} \operatorname{Fix}\left(f^{i}\right)=\left\{z_{2}, \ldots, z_{r-1}\right\}$. Again, by the Kupka-Smale approximation argument, we may assume that $\operatorname{Fix}\left(f^{r}\right)$ is finite. Then the set

$$
A=\operatorname{Fix}\left(f^{r}\right) \backslash\left(\bigcup_{i<r} \operatorname{Fix}\left(f^{i}\right)\right)=\operatorname{Fix}\left(f^{r}\right) \backslash\left\{z_{2}, \ldots, z_{r-1}\right\}
$$

splits into $r$-orbits; hence $\operatorname{ind}\left(f^{r}, A\right)$ is divisible by $r$.

We deform $f$ by the Creating Procedure (again $n=1$ ), rel. a neighborhood of $\left\{z_{2}, \ldots, z_{r-1}\right\}$, to a map $\bar{f}_{r}$ having a fixed point $z_{r}$ such that in its neighborhood

$\bar{f}_{r}=f_{a_{d}}$ with $a_{d}=\frac{\operatorname{ind}\left(f^{r}, A\right)}{r}, d=r$ (cf. item 4 of Theorem 2.3 and (**) of Lemma 2.1).

We will show that $S=\left\{z_{2}, \ldots, z_{r}\right\}$ and that the $\bar{f}_{r}$ satisfy the assumptions of Lemma 2.4. In fact only the condition (3), i.e., $\operatorname{ind}\left(\bar{f}_{r}^{n}, \operatorname{Fix}\left(\bar{f}_{r}^{n}\right) \backslash S\right)=0$ for $n \mid r$, needs to be checked. For $n<r$, ind $\left(\bar{f}^{n}, z_{r}\right)=0$ (cf. (**) of Lemma 2.1); thus

$$
\operatorname{ind}\left(\bar{f}_{r}^{n}, S\right)=\operatorname{ind}\left(\bar{f}_{r}^{n},\left\{z_{2}, \ldots, z_{r-1}, z_{r}\right\}\right)=\operatorname{ind}\left(\bar{f}_{r}^{n},\left\{z_{2}, \ldots, z_{r-1}\right\}\right) .
$$

On the other hand, by item (2) of Theorem 2.3 and homotopy invariance of the fixed point index we get

$$
\operatorname{ind}\left(\bar{f}_{r}^{n},\left\{z_{2}, \ldots, z_{r-1}\right\}\right)=\operatorname{ind}\left(f^{n},\left\{z_{2}, \ldots, z_{r-1}\right\}\right)=\operatorname{ind}\left(f^{n}\right)=\operatorname{ind}\left(\bar{f}_{r}^{n}\right) .
$$


Now $\operatorname{ind}\left(\bar{f}_{r}^{n}\right)=\operatorname{ind}\left(\bar{f}_{r}^{n}, S\right)$, which implies, by additivity of the fixed point index, that $\operatorname{ind}\left(\bar{f}_{r}^{n}, \operatorname{Fix}\left(\bar{f}_{r}^{n}\right) \backslash S\right)=0$, as desired.

For $n=r, \operatorname{ind}\left(\bar{f}_{r}^{n}, z_{r}\right)=\operatorname{ind}\left(f^{r}, A\right)$; thus

$$
\begin{aligned}
\operatorname{ind}\left(\bar{f}_{r}^{r},\left\{z_{2}, \ldots, z_{r-1}, z_{r}\right\}\right) & =\operatorname{ind}\left(\bar{f}_{r}^{r},\left\{z_{2}, \ldots, z_{r-1}\right\}\right)+\operatorname{ind}\left(\bar{f}_{r}^{r}, z_{r}\right) \\
& =\operatorname{ind}\left(f^{r},\left\{z_{2}, \ldots, z_{r-1}\right\}\right)+\operatorname{ind}\left(f^{r}, A\right) \\
& =\operatorname{ind}\left(f^{r},\left\{z_{2}, \ldots, z_{r-1}\right\}\right)+\operatorname{ind}\left(f^{r}, \operatorname{Fix}\left(f^{r}\right) \backslash\left\{z_{2}, \ldots, z_{r-1}\right\}\right) \\
& =\operatorname{ind}\left(f^{r}, \operatorname{Fix}\left(f^{r}\right)\right)=\operatorname{ind}\left(f^{r}\right) .
\end{aligned}
$$

Thus,

$$
\operatorname{ind}\left(\bar{f}_{r}^{r},\left\{z_{2}, \ldots, z_{r-1}, z_{r}\right\}\right)=\operatorname{ind}\left(f^{r}\right)=\operatorname{ind}\left(\bar{f}_{r}^{r}\right) .
$$

As a consequence, $\operatorname{ind}\left(\bar{f}_{r}^{r}, \operatorname{Fix}\left(\bar{f}_{r}^{r}\right) \backslash S\right)=0$, as desired. Then Lemma 2.4 allows us to deform $\bar{f}_{r}$ to a map $f_{r}$ with $\operatorname{Fix}\left(f_{r}^{r}\right)=S=\left\{z_{2}, \ldots, z_{r}\right\}$.

Finally, if $f_{r}$ is not smooth as the self-map of $M$, we approximate it by a smooth map in the same way as we did with $f_{2}$, which ends the proof.

Remark 3.1. A map $g$, homotopic to the given $f$, with the growth of periodic points no faster than linear to a prescribed period $r$, can be chosen as smooth as desired. Namely, we can assure (cf. Remark 4 in GNP) that the maps which realize the indices of iterations from Lemma 2.1 are $C^{l}$, where $l$ is any natural number. This implies that we can find $g$ which is a $C^{l}$ map with $l$ arbitrarily large.

\section{ACKNOWLEDGMENTS}

The authors thank Professor Wacław Marzantowicz for the inspiration, encouragement and helpful comments.

\section{REFERENCES}

[BaBo] I. K. Babenko, S. A. Bogatyi, Behavior of the index of periodic points under iterations of a mapping, Math. USSR Izv. 38 (1992), 1-26. MR.1130026 (93a:58139)

[B] R. F. Brown, The Lefschetz Fixed Point Theorem, Glenview, New York, 1971. MR0283793 (44:1023)

[CMPY] S.-N. Chow, J. Mallet-Paret, J. A. Yorke, A periodic point index which is a bifurcation invariant, Geometric dynamics (Rio de Janeiro, 1981), 109-131, Springer Lecture Notes in Math. 1007, Berlin 1983.

[D1] A. Dold, Fixed point indices of iterated maps, Invent. Math. 74 (1983), 419-435. MR:0724012(85c:54077)

[D2] A. Dold, Lectures on algebraic topology, Springer-Verlag, Berlin, 1995. MR1335915 (96c:55001)

[GNP] G. Graff and M. Nowak-Przygodzki, Fixed point indices of iterations of $C^{1}$ maps in $\mathbb{R}^{3}$, Discrete Cont. Dyn. Syst. 16 (2006), 843-856.

[Je] J. Jezierski, Wecken's theorem for periodic points in dimension at least 3, Topology Appl. 153 (2006), no. 11, 1825-1837. MR2227029

[JM] J. Jezierski and W. Marzantowicz, Homotopy methods in topological fixed and periodic points theory, Topological Fixed Point Theory and Its Applications, Vol 3. Springer, Dordrecht, 2005. MR2189944(2006i:55003)

[K] V. Kaloshin, Generic diffeomorphisms with superexponential growth of number of periodic orbits, Comm. Math. Phys. 211 (2000), no. 1, 253-271. MR1757015 (2001e:37035)

[SS] M. Shub, P. Sullivan, A remark on the Lefschetz fixed point formula for differentialble maps, Topology 13 (1974), 189-191. MR0350782 (50:3274) 
Faculty of Applied Physics and Mathematics, Gdansk University of Technology, Narutowicza 11/12, 80-952 Gdansk, Poland

E-mail address: graff@mif.pg.gda.pl

Institute of Applications of Mathematics, Warsaw University of Life Sciences (SGGW), Nowoursynowska 159, 00-757 Warsaw, Poland

E-mail address: jezierski@acn.waw.pl 